\title{
Modified Pull-out Suture in Posterior Root Tear of the Medial Meniscus: Using a Posteromedial Portal
}

\author{
Jin Ho Cho, $\mathrm{MD}, \mathrm{PhD}$ \\ Department of Orthopaedic Surgery, Inje University Ilsan Paik Hospital, Inje University College of Medicine, Goyang, Korea
}

In cases with root tear of the medial meniscus posterior horn, the meniscus usually can be repaired by a pull out suture technique. However, there is difficulty in manipulating a suture hook via the anteromedial portal and looking through the arthroscopic camera via anterolateral portal in the narrow medial joint space at the same time. This article describes a modified simple pull out suture technique for root tear of the medial meniscus posterior horn using a posteromedial portal that provides a safe and easy handling of the suture hook. Our indications of this technique used in patients with Outerbridge 1-2 arthritic change and minimal varus axis change. Benefits of this technique are simple, less invasive, and reduced operation time by simultaneous suture with a hook via posteromedial portal and pulling of a string with grasper. It may reduce the possibility of an additional chondral or meniscal injury.

Key words: Root tear of posterior horn, Medial meniscus, Modified pull-out suture.

In cases where transection of the posterior horn of the medial meniscus occurs, the ability of the meniscus to withstand hoop tension is destroyed. As a result, extrusion of the medial meniscus from the joint space occurs; this can then lead to the development of a medial compartment degenerative arthritis ${ }^{1,2)}$. Complete radial tear of the meniscus posterior horn, meniscus repair by a pull out suture or suture anchor repair of the meniscus may be used to restore the hoop tension of the meniscus. However, it is technically difficult to handle a suture hook via anteromedial portal and looking at arthroscopic camera via anterolateral portal in the narrow medial joint space at the same time. To solve this problem, we developed a modified simple pull out

Received October 1, 2011; Revised November 6, 2011;

Accepted December 4, 2011.

Correspondence to: Jin Ho Cho, MD, $\mathrm{PhD}$.

Department of Orthopaedic Surgery, Inje University Ilsan Paik Hospital, Inje University College of Medicine, 170 Juhwa-ro, Ilsanseo-gu, Goyang 411-706, Korea.

Tel: +82-31-910-7928, Fax: +82-31-910-7967

Email: osd07@paik.ac.kr

This is an Open Access article distributed under the terms of the Creative Commons Attribution Non-Commercial License (http://creativecommons.org/licenses/by-nc/3.0/) which permits unrestricted non-commercial use, distribution, and reproduction in any medium, provided the original work is properly cited. suture technique for root tear of the medial meniscus posterior horn using a posteromedial portal that provides a safe and easy handling of the suture hook. It may reduce the possibility of additional a chondral or meniscal injury. In addition, it can produce the accurate repair of root tear of the medial meniscus posterior horn easily and reduce operation time.

Here, we describe the simple pull-out suture technique to repair root tear of the posterior horn of a medial meniscus using a posteromedial portal.

\section{Surgical Technique}

A routine arthroscopic examination of the knee joint was performed through the standard anterolateral and anteromedial portals with a distended knee joint at the arthroscopic infusion pump. Using the transillumination technique, a posteromedial portal was made. After confirmation of a lesion (Fig. 1), the footprint was confirmed by removing the cartilage of the tibial plateau, which was attached to the posterior root of the medial meniscus, with a curette and round bur inserted through the posteromedial portal. Under visualization through the anterolateral portal, the anterior cruciate ligament tibial drilling guide (Linvatec, Largo, FL, USA) was introduced through the posteromedial portal. The tip of the guide was placed at the decorticated footprint of the posterior root of the medial meniscus. The sleeve of the guide with $40^{\circ}$ to $45^{\circ}$ was fixed on the 
anteromedial cortex of the proximal tibia after a 2 -cm vertical incision was made.

The entry point of tibial anteromedial cortex aimed midportion of tibial shaft near 2 to $3 \mathrm{~cm}$ anterior portion from medial collateral ligament insertion. A guide pin was drilled from the anteromedial cortex of proximal tibia to the insertion site of

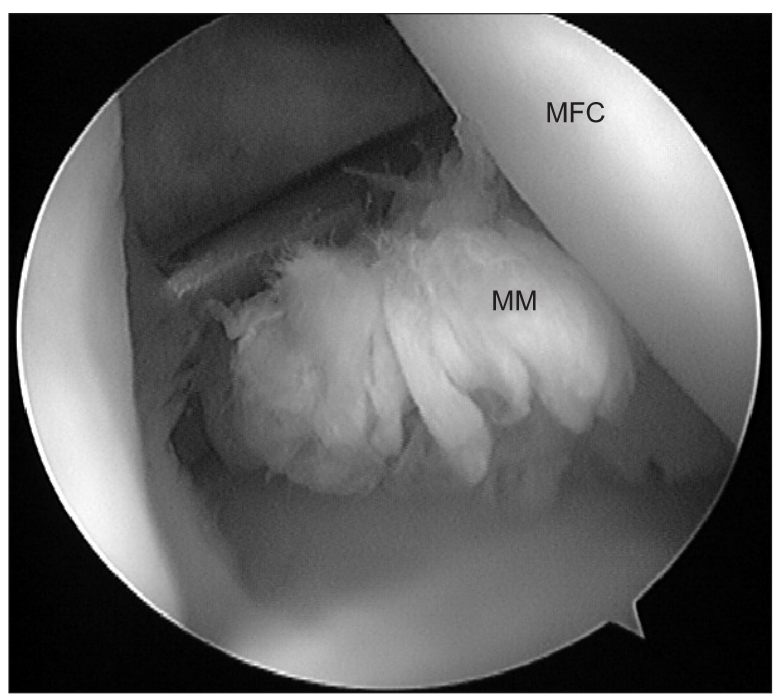

Fig. 1. Arthroscopic photograph shows the complete root tear of the posterior horn of the MM through anterolateral portal of the right knee, which was displaced completely by a probe inserted through the anteromedial portal. MM: medial meniscus, MFC: medial femoral condyle.

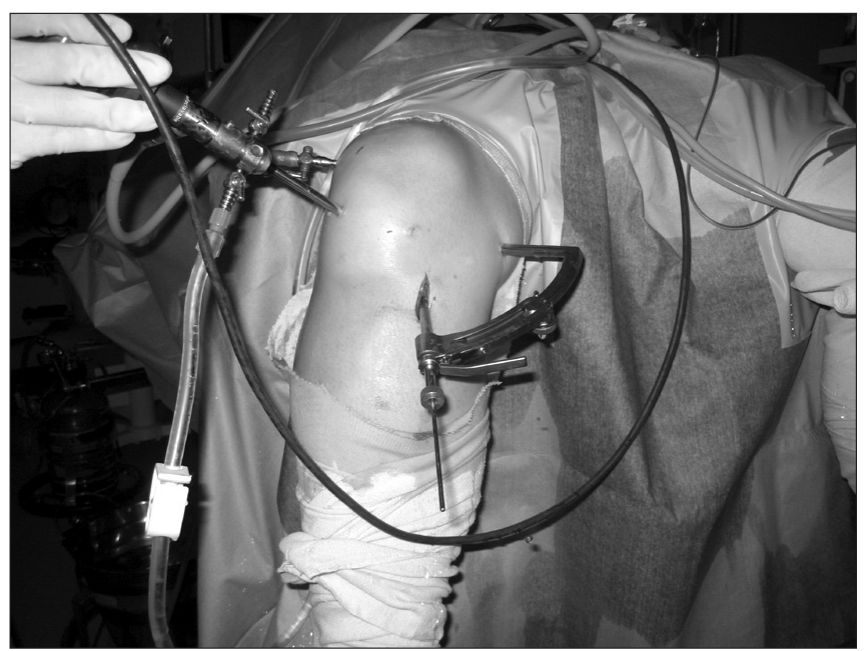

Fig. 2. Under visualization through the anterolateral portal, the anterior cruciate ligament tibial drilling guide was introduced through the posteromedial portal. The sleeve of the guide was fixed on the anteromedial cortex of the proximal tibia after a 2 -cm vertical incision was made. A guide pin was drilled from the anteromedial cortex of the proximal tibia to the insertion site of the posterior root tear site of the medial meniscus through the sleeve. the posterior root tear site of the medial meniscus through the sleeve (Fig. 2). A tibial tunnel was made from the anteromedial cortex of the proximal tibia to the footprint of the posterior root of the medial meniscus by use of a guide pin with a $6 \mathrm{~mm}$ reamer (Linvatec). While visualizing from anterolateral portal, a crescent-shaped suture hook (Linvatec) loaded with a No. 0

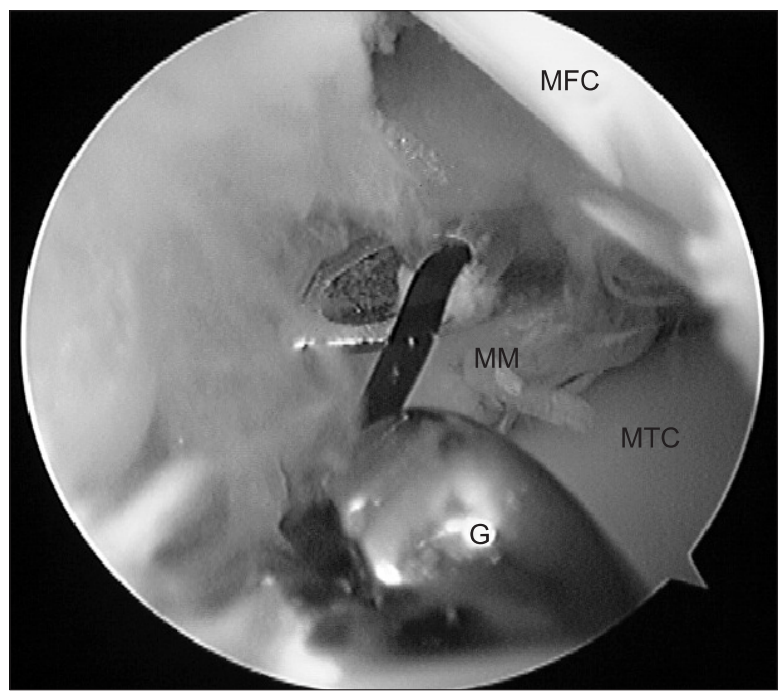

Fig. 3. This arthroscopic photograph shows the sharp tip of the crescentshaped suture hook penetrating the detached root portion of the MM posterior horn from the femoral surface to the tibial surface in a vertical direction. After one end of the strands of the PDS was advanced through the suture hook, it was retrieved out through transtibial tunnel with already inserted grasper at the same time. MM: medial meniscus, MFC: medial femoral condyle, MTC: medial tibial condyle, G: grasper.

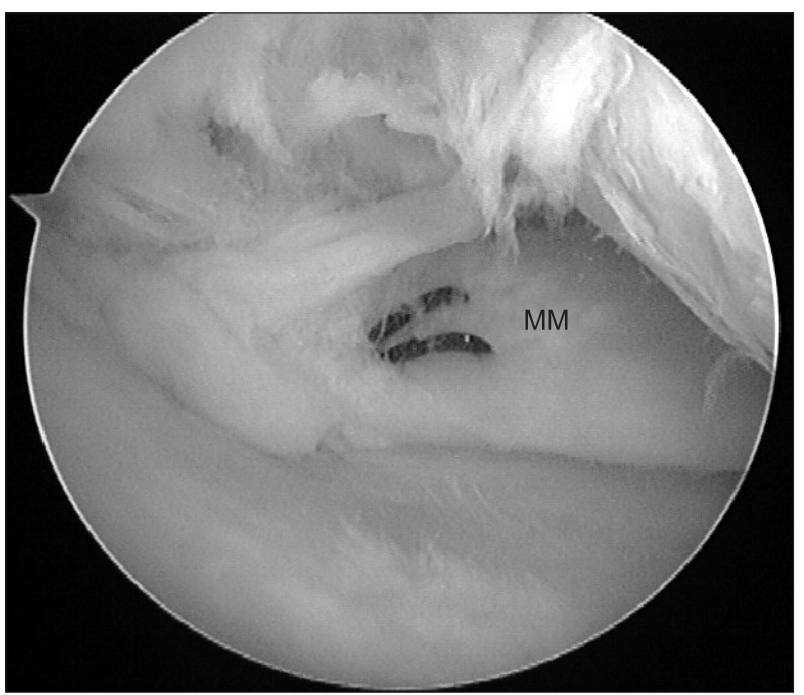

Fig. 4. Arthroscopic photograph from the anterolateral portal shows reattachment of the posterior root of the medial meniscus to the tibial tunnel site in adequate tension. MM: medial meniscus. 
PDS (Ethicon, Somerville, NJ, USA) suture material was inserted through the posteromedial portal. The detached root portion of the medial meniscus posterior horn was penetrated by the sharp tip of the crescent-shaped suture hook from the femoral surface to the tibial surface of the meniscus in a vertical direction. Then No 0 PDS suture was advanced through the suture hook. After passing some of the total length of the PDS, already inserted grasper, it was advanced into the intra-articular space through the tibial tunnel, retrieved out one end of the strands of the PDS through tibial tunnel at the same time (Fig. 3). Such simultaneous procedure can save operation time. Then the suture hook was upward withdrawn. Then, the other ends of the PDS strands were retrieved through a tibial tunnel with a grasper again. In this manner, additional 1 PDS suture material was sutured through the posterior horn of the medial meniscus out into the tibial tunnel with grasper. By pulling the ends of the suture materials under adequate tension, the posterior root of the medial meniscus could be reduced and stabilized with adequate tension (Fig. 4). Under adequate reduction and tension, the PDS suture materials were post-tied to and fixed with a $6.5 \mathrm{~mm}$ cancellous bone screw and smooth washer on the anterior cortex of the tibia.

\section{Postoperative Management}

After surgery, a cylinder leg splint was applied for 2 weeks with the leg in a fully extended position and a limited-motion brace was subsequently applied to control motion. Postoperatively, patients were non-weight bearing on crutches for 6 weeks. Patients were introduced to perform quadriceps muscle exercise, as well as straight-leg raise exercises several times daily. Patients were allowed active motion of up to $90^{\circ}$ after the first 4 weeks. Then, patients were allowed to gradually increased flexion degrees up to $135^{\circ}$. Full flexion and squatting were allowed 3 months after the surgery. Patients returned to exercise after 6 months postoperative. We performed 13 cases (65\% in total 20 cases) of second look arthroscopy and screw removal. We checked well healed repaired site and improved patient's symptoms.

\section{Discussion}

Bin et al. ${ }^{3)}$ examined 345 consecutive knee arthroscopies performed to treat a medial meniscus tear and found that $28 \%$ of all medial meniscal tears involved the posterior attachment site. Of those for which magnetic resonance imaging (MRI) scans were available, meniscal extrusion was present in $79 \%$ of patients with complete medial meniscal root tears, in $64 \%$ with partial medial meniscal root tears, and in 50\% with a degenerative medial meniscal root.

The patient may feel a click or have a sensation of giving way, associated with medial joint pain. Physical examination findings usually consist of tenderness at the medial joint line, a minor effusion, and slightly diminished knee flexion ${ }^{3)}$. The McMurray test is positive for pain with varus stress, but this does not create mechanical click. Meniscal extrusion on MRI scan, defined as $>3 \mathrm{~mm}$ measured from the outer margin of the meniscus to the outer articular margin of the medial tibial plateau on mid coronal MRI scans ${ }^{4)}$ is an important secondary sign that there may be pathology at the meniscal root origin.

In the past, some authors treated posterior horn meniscus root tear using a partial meniscectomy. However, it has been recognized that while some of these patients do well, many patients continue to have knee pain with weight-bearing activities and are dissatisfied with the outcome of this procedure. Partial meniscectomy does not restore the normal biomechanical function of the meniscus. It is believed that a medial meniscus root tear should be repaired to recover hoop tension, even though the posterior horn attachment of the meniscus is covered with vascular synovial tissue and appears to have a good blood supply ${ }^{5)}$.

Surgical options include partial meniscectomy, meniscal repair, and meniscal transplantation. Repair is a reasonable option in acute and subacute cases or in chronic cases in which there is little or no articular cartilage disease and good preservation of healthy meniscal tissue. Several techniques have been reported in the literature, Raustol et al. ${ }^{6)}$ used a long pin and sutures passed through a posteromedial accessory arthroscopy portal in a transosseous fashion. Recently, Griffith et al. ${ }^{7)}$ described a technique that combines an arthroscopic approach to repair the medial meniscal root to a decorticated footprint via suture tunnels over a bone bridge. Ahn et al. ${ }^{8)}$ reported pull out suture using a posterior trans-septal portal.

We used all-arthroscopic modified pull-out suture technique in an attempt to restore the attachment. Benefits of this technique are simple, less invasive, and reduced operation time by simultaneous suture with a hook via posteromedial portal and pulling of the string with a grasper. Our indications of this technique were patients with Outerbridge 1-2 arthritic change and minimal varus axis change.

We think that this procedure is very simple and less invasive. This technique minimizes iatrogenic injury to the cartilage and saves operation time. 


\section{References}

1. Kenny C. Radial displacement of the medial meniscus and Fairbank's signs. Clin Orthop Relat Res. 1997;(339):163-73.

2. Lerer DB, Umans HR, Hu MX, Jones MH. The role of meniscal root pathology and radial meniscal tear in medial meniscal extrusion. Skeletal Radiol. 2004;33:569-74.

3. Bin SI, Kim JM, Shin SJ. Radial tears of the posterior horn of the medial meniscus. Arthroscopy. 2004;20:373-8.

4. Jones AO, Houang MT, Low RS, Wood DG. Medial meniscus posterior root attachment injury and degeneration: MRI findings. Australas Radiol. 2006;50:306-13.

5. Arnoczky SP, Warren RF. Microvasculature of the human meniscus. Am J Sports Med. 1982;10:90-5.

6. Raustol OA, Poelstra KA, Chhabra A, Diduch DR. The meniscal ossicle revisited: etiology and an arthroscopic technique for treatment. Arthroscopy. 2006;22:687.e1-3.

7. Griffith CJ, LaPrade RF, Fritts HM, Morgan PM. Posterior root avulsion fracture of the medial meniscus in an adolescent female patient with surgical reattachment. Am J Sports Med. 2008;36:789-92.

8. Ahn JH, Wang JH, Yoo JC, Noh HK, Park JH. A pull out suture for transection of the posterior horn of the medial meniscus: using a posterior trans-septal portal. Knee Surg Sports Traumatol Arthrosc. 2007;15:1510-3. 